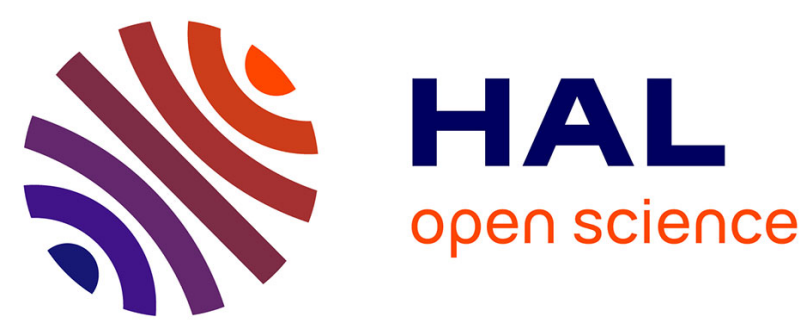

\title{
ATOMIC AND MOLECULAR AUTOIONIZING STATES. A THEORETICAL APPROACH
}

\author{
A. Macias, F. Martin, O. Mo, A. Riera, M. Yañez
}

\section{To cite this version:}

A. Macias, F. Martin, O. Mo, A. Riera, M. Yañez. ATOMIC AND MOLECULAR AUTOIONIZING STATES. A THEORETICAL APPROACH. Journal de Physique Colloques, 1989, 50 (C1), pp.C1-37-

C1-52. 10.1051/jphyscol:1989105 . jpa-00229295

\section{HAL Id: jpa-00229295 https://hal.science/jpa-00229295}

Submitted on 1 Jan 1989

HAL is a multi-disciplinary open access archive for the deposit and dissemination of scientific research documents, whether they are published or not. The documents may come from teaching and research institutions in France or abroad, or from public or private research centers.
L'archive ouverte pluridisciplinaire HAL, est destinée au dépôt et à la diffusion de documents scientifiques de niveau recherche, publiés ou non, émanant des établissements d'enseignement et de recherche français ou étrangers, des laboratoires publics ou privés. 
JOURNAL DE PHYSIQUE

Colloque $C 1$, supplément au $n^{\circ} 1$, Tome 50 , janvier 1989

ATOMIC AND MOLECULAR AUTOIONIZING STATES. A THEORETICAL APPROACH

\author{
A. MACIAS, F. MARTIN, O. MO, A. RIERA and M. YAÑEZ \\ Departamento de Quimica, C-XIV Universidad Autonoma de Madrid, \\ Cantoblanco, SP-28049 Madrid, Spain
}

\begin{abstract}
Resume - Nous presentons une nouvelle methode qui permet l'obtention des positions et durees de vie des etats resonants, atomiques ou moleculaires, avec deux electrons actifs. Cette procedure, est basee sur une analogie formelle entre le formalisme de Feshbach et la methode du pseudopotentiel de Phillips et Kleinman. Elle produit des resultats en tres bon accord avec ceux de la méthode de Feshbach avec un calcul beaucoup plus simple, spectalement pour le cas où I'etat resonant se trouve au dessus de plusieurs seulls d'ionisation. Cette methode peut etre generalisée facilement au traitement des etats doublement excites des systemes

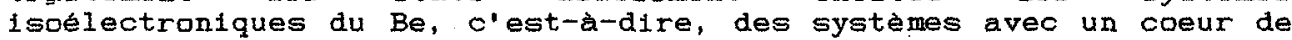
couche fermee 1s\%. Dans notre application de la methode la fonction d'onde de la composante ouverte s'obtient en utilisant une technique de discretisation du continu, dont les bases sont présentees en detail. On utilise aussi une methode d'interpolation inverse pour remplir la condition de degeneration discret-continu qui est necessaire pour appliquer la régle d'or et obtenir la largeur. Nous presentons quelques illustrations pour des états résonants de systemes Heliumoide et Berylliumoide qui sont au dessus de plusieurs seuils d'ionisation et aussi pour des résonances moleculaires de systemes homo et heteronucleaires deux electrons.
\end{abstract}

\begin{abstract}
We present a progress report on a new method to calculate positions and widths of two-electron atomic (molecular) resonances This procedure, based on a formal analogy between the Feshbach formalism and the Phillips-Kleinman pseudopotential approach, yields results in very good agreement with those obtained with the standard Feshbach formalism, but requires a considerably smaller computational effort in cases where the resonant states of interest lie above several ionization thresholds. This method can be very easily generalized to the treatment of doubly excited states of Belike systems, that is containing a closed shell 1s: core. In our implementation of the method, the open channel wavefunction is obtained from using a discretization procedure, whose theoretical basis we present in some detall, and an inverse interpolation method to achleve the discrete-continuum degeneracy which is needed to aply the golden rule formula to calculate the width. We present some illustrations for He-like and Be-like resonances lying above one or several ionization thresholds, and for molecular resonances of homo and heteronuclear two-electron systems.
\end{abstract}

\title{
1 - INTRODUCIION
}

Doubly excited states of atoms and molecules have received a great deal af attention because they are involved in a considerable number of experimental phenomena such as beam-foll spectroscopy $/ 1,2 /$, electronimpact experiments /3/, atomic processes in fusion plasmas /4/, and siglecollision beam experiments $/ 5-12 /$.

One of the most powerful tools to obtain the positions and widths of this kind of resonances is the well known Feshbach formalism /13/. whose fundamental definitions are sumarized in section 2 . In this approach the 
wavefunction representing the autolonizing state of a (formal) two-electron system is written as the sum of a closed channel, $Q Y$, and an open channel Py, components. Solutions of the closed channel equation have been reported for He-like resonances lying above the first ionization limit /14-18/; on the other hand, results for resonances which are above several ionization thresholds are scarce /19/, because the method becomes computationally cumbersome. Likewise, only very recently theoretical results for (1s: $n l n^{\prime} l^{\prime}$, resonant states of Be-like systems have been reported $/ 20 /$ in the Iiterature and there is a complete lack of theoretical information on $\left(1 \operatorname{sn} 1 n^{\prime} I^{\prime}\right)$ resonances of Li-11ke systems, because, although these states are of an increasing interest, the Feshbach approach is extremely difficult to implement for systems with more than two electrons.

We present in this paper a progress report on an alternative method we have recently proposed that yields positions and widths $/ 21,22 /$ in very good agreement with those obtained with the Feshbach formalism while requiring a considerably smaller computational effort. Our method is based $/ 23 /$ on a formal analogy between the Feshbach theory and the Phillips-Kleinman pseudopotential approach $/ 24 /$ to be described in Section 3 . We shall show in Section 4 that this procedure constitutes a general method which permits to obtain, through an adequate definition of this pseudopotential, either the energies of the valence states of species with a closed-shell core or the positions of autoionizing states of systems with or without a core and lying above one or several ionization thresholds.

To approximate the open channel wavefunction and calculate its interaction with the corresponding closed channel component, we have developped a discretization procedure $/ 25 /$ whose theoretical basis will be summarized in section 5 . The calculation of the width will be explained in section 6 and in section 7 we shall present some 11 lustrations for He-Iike /26/ and Be-like /20/ resonances lying above several ionization thresholds and discuss the extension of this theoretical treatment to the study of molecular resonances $/ 27 /$.

\section{2 - EESHBACH THEORY FOR TWO-ELFCTRON SYSTEMS}

In order to better understand the new theory presented in section 4 , it is worth to recall the basic definitions of the Feshbach formalism $/ 13 /$.

In this theory the wave function representing a two-electron resonant state is written as a sum of open and closed channel components:

$$
\psi\left(\mathbf{r}_{1}, \mathbf{r}_{2}\right)=P \psi+Q \psi
$$

where $P$ is a projection operator such that $P y$ behaves asymptotically ilke $\psi$ when either $r_{7}$ or $r$ e tends to infinity; and $Q=1-P$. For two-electron systems the O'Malley-Geltman form /28/ for $Q$ is generally used:

where

$$
Q=Q, Q
$$

$$
Q_{*}=1-P_{i}=1-\Sigma \times\left|\varphi_{\alpha}(i)\right\rangle\left\langle\varphi_{\alpha}(i)\right|=1-\Sigma \alpha P_{\alpha}(1)
$$

and where the sum in eq. (3) runs over the Nc threshold states, whose energles lie below the doubly excited state considered, and are defined by the hydrogento orbitals $\varphi_{x x}$.

Inserting eq. (1) in the Schrödinger equation yields the coupled equations:

$$
\begin{aligned}
& \left(H_{Q Q}-E\right) Q \psi=-H_{Q F} P Y \\
& \left(H_{F F}-E\right) P \psi=-H_{Q} Q \psi
\end{aligned}
$$

where $\mathrm{HQQ}_{Q}=\mathrm{QHQ}_{\mathrm{Q}} \mathrm{HFF}=\mathrm{PHP}$ and $\mathrm{HFQ}_{\mathrm{F}}{ }^{+}=\mathrm{HQF}_{\mathrm{O}}=\mathrm{PHQ}$.

The projected Hamiltonian Haq has a discrete spectrum below the Nat1 ionization limit and the interaction $H_{\alpha F}$ permits the decay of the closed channe1 QY into the continuum state PY.

Then, the closed channel wavefunction is obtained as the elgenfunction of:

$$
\left\langle\mathrm{H}_{\mathrm{Q} Q \Omega}-E_{n} \boldsymbol{}\right) \phi_{n}=0
$$

When more than one open channel is open we can define the partial width corresponding to each Lonization threshold, $\alpha$, as: 


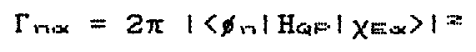

where the corresponding open channel component $\chi E x$ is (neglecting terms quadratic in $\left.H_{a P}\right)$ the eigenfunction of the equation:

$$
\left(P_{1 x} H_{P x}-E^{e}\right) X E_{1 x}=0
$$

for $E^{E}=E_{10}$ and

$$
P_{x}=P_{x x}(1)+P_{x x}(2)-P_{x x}(1) P_{x x}(2)
$$

The total width is given by /13/:

$$
\Gamma_{n}=\Sigma_{\alpha} \Gamma_{\text {rax }}
$$

In the $\left\{\phi_{n}, X_{\alpha}\right\}$ representation, the Hamiltonian can be symbolically written as:

$$
H=\left(\begin{array}{ll}
\mathbf{E}^{\sigma} & \mathbf{H}_{\sigma_{F}} \\
\mathbf{H}_{F Q} & \mathbf{E}^{c}
\end{array}\right)
$$

where $\mathrm{B}^{\mathrm{a}}$ and $\mathrm{E}^{\mathrm{c}}$ are diagonal matrices. As indicated above, the elements of E are discrete below the $N_{c}+1$ threshold, and correspond to the closed channel energies of Eq. (5). The $\mathrm{E}$ matrix contains a discrete part, which corresponds to the Rydberg series converging to the first lonization limit and an open channel continuum part, which corresponds to the ionization continua.

In practice, the closed channel equation (eq. (5)) is usually solved by using either:

a) the exact solutions of a separable Hamiltonian such as:

$$
H^{\circ}=H_{-}-r_{1} z^{-1}=H^{\infty}\langle 1\rangle+H^{\circ}\langle 2\rangle
$$

as a basis set, or

b) a standard CI method, with configurations built from slater or Gaussian-type atomic orbitals that have been orthogonalized to the set $\left\{\varphi_{x}\right\}$ in Eq, 〈3〉.

Both implementations become considerably complicated when more than one ionization threshold is open: in a) because of slow convergence and substantial programing complication; in b) because the orbital basis used to build the configurations must be simultaneously orthogonalized to the different sets $\left\{\varphi_{x}\right\}$ involved in the definition of $Q$, which makes the procedure very difficult to apply.

\section{3 - EESHBACH THEORY EOR SYSTEUS WITH A CLOSED-SHELI CORE}

Let us consider now the particular case of the resonant states of a system with a closed-shell core. To be specific, let us assume that we are interested in the (1s: 3131 ') autoionizing states of Be-like systems. As indicated in the Introduction, the Feshbach formalism is extremely difficult to implement for a four-electron system. On the other hand, we can take into account that the core $1 s^{2}$ electrons play an almost passive role, and use a model potential /29/ to account for the core-valence effects. This provides a practical alternative to the full-electron treatment, since it reduces the problem to a formal two-electron case, and the Feshbach formalism outlined in the previous section can be applied.

In this approach we replace the four-electron Hamiltonian by a model one of the form:

$$
\mathrm{H}_{M}=\mathrm{H}^{\circ}(1)+\mathrm{H}^{\circ}(2)+\mathrm{V}_{M}(1)+\mathrm{V}_{M}(2)+\mathrm{r}_{1} \mathrm{M}^{-1}
$$

where $H^{\circ}$ is a hydrogenic Hamlitonian and $V_{m}$ a suitable model potential /30$32 /$ that accounts for the core-valence effects. We can then carry out a variational calculation for $\mathrm{H}_{m}$ solving the corresponding secular equation in a representation of configurations of the form:

$$
\psi_{k i, k}=1 / 2\left[\varphi_{k i}(1) \varphi_{k},(2) \pm \varphi_{k:},(1) \varphi_{k:(2)]}\right.
$$


where $\varphi_{\text {r }}$ are one-electron wavefunctions and the spin functions have been factored out for simplicity. A well-known /30-32/ peculiarity of model potential calculations is that the valence states of the system are not described by the lowest eigenvalues of (12), but are, from a formal paint of view, infinitely excited. This is because, below the valence states (2s:2, 2s2p, $2 p ; \ldots$ ) of the system there exists a infinite number of unphysical "core" or "virtual" states, which belong to the unphysical

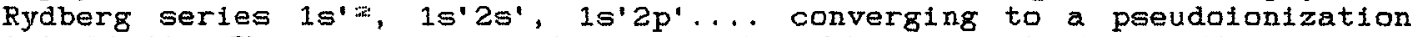
1s' Iimit. The consequence is that, in this approximation, the valence states of this atomic system will lie above this pseudaionization threshold and are therefore embedded in the virtual continuum of the model Haml Itonian.

We notice that the previous feature of the simplified theory implies that in this model there is no difference whatsoever between the two-electron valence states (1s:2121') and the ( $1 \mathrm{~s}^{2} 3131$ ') autoionizing ones. Actually the only difference is that while the latter lie above several fonization thresholds (1s', $2 s$ and $2 p$, one of which is non-physical) the former 11 )s above a single (15') threshold. This difference becomes even less significant if we consider a system as $\mathrm{Mg}$ where the two-electron valence states would lie above several pseudaionization (15', $2 s^{\prime}$ and $2 \mathrm{p}^{\prime}$ ) thresholds.

We see then that the model potential approach provides an analogy /23/ between doubly excited resonant and two-electron valence states. As a consequence, the latter can be obtained with the same methodology as the former, e.g., by solving an equation similar to Eq. (5). This would also mean that the difficulties indicated at the end of section 2 to implement the Feshbach formalism for resonances lying above several ionization thresholds will also apply here.

\section{4 - EESHBACE-PSEUDOPOTENTIAL APPROACH}

In the model potential approach, there is however a simpler way to calculate the valence energies which consists in adding to the model Hamiltonian (12) a level shift operator or pseudopotential Ves,

$$
\mathrm{H}_{\text {w }}+\mathrm{H}^{\prime}=\mathrm{H}_{\mathrm{m}}+\mathrm{V}: \mathrm{V}
$$

so that the lowest elgenvalue of $H_{w i}+$ corresponds to the two-electron state of interest. According to our analogy, this way can be also applied /21, 22/ to obtain the resonance energies of doubly excited states.

To see the implementation of the method let us consider again, for instance, a Be-like system. We can chose $v_{p}$ as a generalization of the Philitps-Kleinman projection $/ 24 /$ operator:

$$
\begin{aligned}
V_{F: s}= & \mid 1 s^{\prime}(1)>M\left\langle 1 s^{\prime}(1)|+| 1 s^{\prime}(2)>\mathbf{M}\left\langle 1 s^{\prime}(2)\right|\right. \\
& -11 s^{\prime}(1) 1 s^{\prime}(2)>M<1 s^{\prime}(1) 1 s^{\prime}(2) \mid
\end{aligned}
$$

which takes the form of a sum of one-electron pseudopotentials, minus a correction term which prevents states with two electrons in the core to be shifted twice, and where $M$ is a suitable large positive number Introduction of this pseudopotential in $H_{*} \cdot$ shifts up the energies of all virtual core states, so that the first eigenvalue of the effective Hamiltonian corresponds to the ground valence state of the system.

on. the other hand, if one is interested in doubly excited states of the system lying above the $2 s$ and $2 p$ thresholds, one can generalize Eq. (15) and write:

where

$$
V_{\mathrm{F}: s}=\mathrm{MP}
$$

$$
\begin{aligned}
P & =\Sigma_{x \times}\left|\varphi_{\alpha}(1)\right\rangle\left\langle\varphi_{x}(1)\left|+\Sigma_{E}\right| \varphi_{E}(2)\right\rangle\left\langle\varphi_{E}(2)\right| \\
& -\Sigma\left|\varphi_{x x}(1) \varphi_{E}(2)\right\rangle\left\langle\varphi_{\alpha}(1) \varphi_{B}(2)\right|
\end{aligned}
$$

is just the projection operator as defined by $P=1-Q$ and Eqs. (2), (3) and the summations involve the 15', $2 s$ and $2 p$ ionization thresholds. Then the first eigenvalue of Eq. (14) corresponds to the lowest (1s 3131 ') autoionizing state. In summary, we may adopt for our pseudopotential operator the form of Eqs. (16) and (17) where the summations in (17) run over as many thresholds as needed depending on the state of interest. 
Comparison of Eq. (16) with Eqs, (3), (5) shows that the effective Hamiltonian can be written in the $\left\{\phi_{n}, X_{m}\right\}$ representation in the following symboltc form:

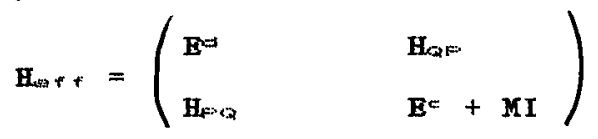

We notice the parallelism between this symbolic matrix and that obtained within the Feshbach-0.Malley theory (See Eq. (10)). However, the most important fact is that the practical solution of the closed channel equation now becomes quite simple because it is no longer necessary to explicitly construct the $Q$ projection operator, and a CI calculation can be carried out directly for the effective Hamiltontan (14). Moreover, the goodness of the approximation will only depend on the quality of the basis set used $/ 23 /$.

Finaliy it should be notlced that this procedure can also be applied to resonant states of two-electron systems lying above several ionization thresholds. It suffices to set $V_{m} \equiv 0$ in Eq. (12).

\section{5 - DISCRETIZATION FOR THE CONTINUUM PROJECTION OF THE VAVEFUNCT ION}

To solve the open channel equation, Eq. (7), we follow the work of Refs 16-18, 25-27 and 33/. We take into account that, from Eq. (6), the form of the open channel component, $X$ ex, is only needed in the region of configuration space where the closed channel component, $\phi_{n}$, which is degenerate with it is non-negligible. This allows us to employ an Lzintegrable representation of $x E$ in this region. Let us assume for the sake of simplicity that we are in a single continuum, so that we can drop the subindex $\alpha$ from our notation.

To obtain the $L=$ representation of $X E$ we can diagonalize HFe in a finite basis of Li-integrable wave functions belonging to the complement of $Q$ subspace. To avoid mathematical complications derived from the fact that $x$ does not belong to a Hilbert space, and is normalized according to Diracts delta function

$$
\left\langle X E \mid X_{E-1}\right\rangle=\delta\left(E-E^{\prime}\right\rangle
$$

we follow the procedure of Macias and Riera /16-18/ and enclose the whole system in a very large box, so that the new eigenfunctions of $H_{F}$. hereafter called $x$, are identical to $x \in$ inside a very large domain of configuration space. Under these conditions the espectral resolution for the projected Hamiltontan is given by:

$$
H_{F: F}=\Sigma_{i}\left|X_{E x}\right\rangle E_{ \pm}\left\langle X E_{1}\right|
$$

Then, we may consider any arbitary function of $X^{k}$ as, for instance, the bracketed expression $\langle f|x|\rangle_{\text {; }}$ while this term is a function of the continuous variable $E$, the corresponding expression $\langle f \mid x \in \pm\rangle$ takes values for discrete $E_{L}$ only and may be represented by a histogram. However, our discretization is dense enough (say energy separation $\mathcal{O}(\epsilon)$ ) for the energy differences to be so small that the histogram may be replaced by a continuous interpalation function. For example, our discretization will replace the normalization (19) by:

$$
\left\langle X_{E=1} \mid X_{E:}\right\rangle=\delta_{*:}\left(E, E^{\prime}\right\rangle
$$

where the interpolation function $\delta_{a}\left(E, E^{\prime}\right)$ is a member of a $\delta$-family, which tends to $\delta\left(\mathrm{E}^{\left.-E^{\prime}\right)}\right.$ as $\epsilon \rightarrow 0$. It is convenient to choose the area under this S. as

$$
\int \delta E^{\prime} \cdot \delta_{:}\left(\mathrm{E}, \mathrm{E}^{\prime}\right)=1
$$

we have also by construction

$$
\delta_{i:}\left(E_{i .}, E_{j}\right)=0 \text { when }\left|E_{i}-E_{j}\right|>E
$$


The advantage of introduoing the fine grained discretization ( 20 ) will now be apparent, because $X=1$ can be approximated, as well as desired, by a basis expansion, which is not the case for $x \in$.

Let us consider now a practical situation in which we diagonalize HFF in a basis set of La integrable functions:

$$
\left\{f_{s} ; j=0,1, \ldots \ldots N\right\}
$$

which is complete in $P$ space as $N \rightarrow \infty$. Its spectrum is formed by a discrete set of eigenvalues $E_{m}$ and each eigenfunction will be giver by:

$$
X_{n}=\Sigma_{f} \operatorname{con} f_{3}
$$

where the coefficients $c_{i n}$ are obtained by solving the corresponding secular equation:

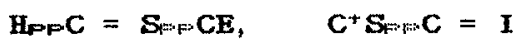

From Eq. (26) the $x_{n}$ wave functions are normalized according to the delta of Kronecker;

$$
\left\langle X_{m} \mid X_{m}\right\rangle=\delta_{m n}
$$

Since the $x=x$ wavefunctions belong to the Hilbert space of $P$ functions and can be expanded in the complete set (24), in the limit $N \rightarrow \infty$ the eigenfunctions $X_{n}$ and $x_{e n}$ must coincide up to a normalization factor

$$
X_{n-1} \rightarrow \delta^{-1} \rightarrow\left(E_{n}, E_{n-1}\right) \quad X E \quad \text { as } \mathbb{H} \rightarrow \infty
$$

so that Eq. (27) holds

$$
\begin{aligned}
& \left\langle\chi_{n} \mid \chi_{m}\right\rangle \underset{N \rightarrow \infty}{\rightarrow} \delta^{-1}-2\left\langle E_{n}, E_{m}\right\rangle \delta^{-1}>2\left\langle E_{m}, E_{m}\right\rangle\left\langle X_{E=n} \mid \chi_{E[m}\right\rangle \\
& =\delta^{-1}-2\left(E_{n}, E_{m}\right) \delta^{-i-2}\left(E_{m}, E_{m}\right) \delta\left(E_{m}, E_{m}\right)=\delta_{m m}(29)
\end{aligned}
$$

If we take now the Heisenberg transform of $x_{m}$

$$
\begin{aligned}
& \left\langle X X_{i} \mid X_{n}\right\rangle \quad \rightarrow \quad \delta_{e^{-1}}^{-1}=\left\langle E_{n}, E_{n}\right\rangle\left\langle X E_{1} \mid X_{E_{n}}\right\rangle=
\end{aligned}
$$

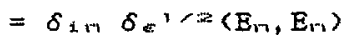

we find that $\left\langle\chi_{E} \mid \chi_{m}\right\rangle$ is completely concentrated about $E_{m}$, 1 , e, for large enough basis, $X_{n}$ has a maximum overlap with the continuum wavefunction $x_{\text {sen }}$ It has also been proved /34/ that this is so, to a good approximation, for smaller basis sets. This is equivalent to write:

$$
X n \simeq p^{-\cdots 1<z}\left(E_{n}\right) X E n
$$

We further notice that formal discretization of the continuum spectrum of $\mathrm{H}_{F}:$ substitutes the projection aperator:

$$
P=\int d E|X| X|X|=1
$$

by the quadrature:

$$
P=\Sigma_{i} \Delta E_{i}\left|X_{E}\right\rangle\left\langle X_{E}\right|
$$

For a complete set, condition (28) is fulfilled and we can write:

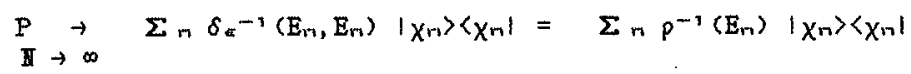

and, since the spectra associated to $\left\{\chi_{\mathrm{E}}\right\}$ and $\left\{\chi_{\mathrm{W}}\right\}$ are identical in the $11 \mathrm{mit} N \rightarrow \infty$, Eqs. (31) and (33) Show that $p\left(E_{w}\right.$ ) may be taken as a density of states

$$
p\left(E_{n}\right) \simeq \Delta n / \Delta E_{n}
$$


As a consequence of the relationship (30) in this discretization approach the golden rule formula becomes:

$$
\Gamma=2 \pi \rho\left(E_{m}\right\rangle\left|\left\langle\phi,\left|H_{G=1}\right| \chi_{n}\right\rangle\right|=
$$

for $E_{n}=E_{1}=$.

The problem now is how to evaluate this density of states. To illustrate our procedure /25-27,33/ let us consider a particular case, supposing that we diagonalize $H$ in a basls of configurations built from even-tempered sequences of STO's or GTO's

$$
\left\{f_{s}\langle\eta\rangle ; j=0,1 \ldots \ldots, N\right)
$$

depending upon a non-linear parameter $\eta$ such that the exponent sequence is

$$
\alpha_{ \pm}\left\langle\eta=\alpha_{1} \text {, क. } \beta=\alpha_{0} \beta+3, \quad 1=0,1, \ldots \ldots, N\right.
$$

To a good approximation the corresponding eigenenergies are the sum of a bound electron energy, $\mathrm{E}^{\circ}$, and the kinetic energy of the "continuum" electron:

$$
E_{n}=E^{\circ}+\tau_{n}\langle\eta\rangle
$$

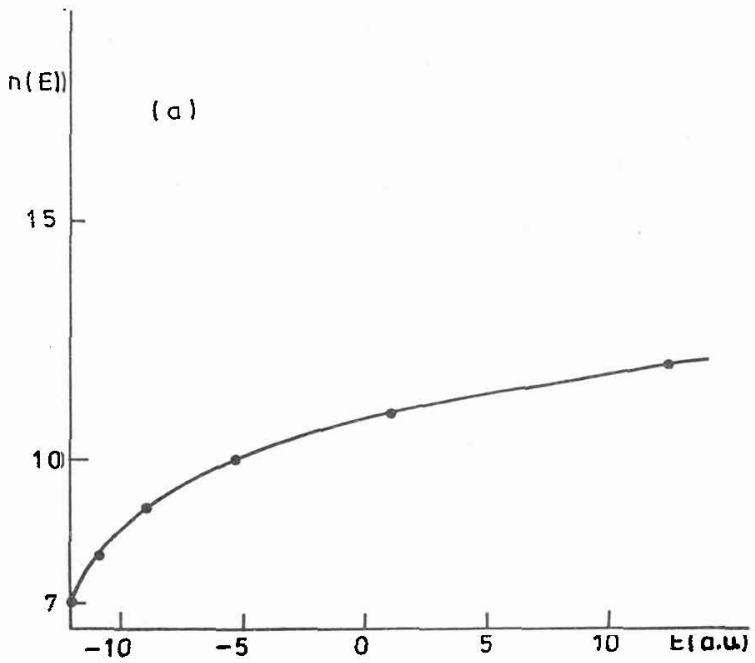

Fig. 1 - Exponential behavior of the eigenvalues of HFF, En as a function of the index $n$.

and present a clear exponential pattern as functions of the index $n$ (See Fig. 1). Actually, it is easy to show $/ 25 /$, from the homogeneous property of the kinetic-energy operator, that these eigenvalues fulfill the equation:

$$
E_{1 \cdots+m}=\tau_{w}(0) e^{x m}+E^{0}
$$

and that, to a very good approximation $/ 25,33 /$, the density of states can be evaluated by the very simple expression:

$$
p\left(E_{n+1}\right)=2 /\left(E_{n+1}-E_{n-1}\right)
$$

Furthermore, the accuracy of this procedure can be gauged by two very simple tests:

1) Results obtained with two different basis sets (1.e. different values of the parameter $\eta$, should yield the same results, to a good precision.

2) Convergence of $\Gamma$ with the size of the basis set $N$ should have been reached to the desired precision. 


\section{6 - IHE CALCULATION OF $\Gamma$}

As stated in the previous section, we approximate the width corresponding to the ionization into a given continuum of the resonant state by Eq. (36) However, it is clear that, in general, none of the eigenvalues of HF. obtained by solving the corresponding secular equation (26) colncides with $E_{\mathrm{A}}{ }^{*}$, unless some additional condition 15 imposed. In our procedure this is easily done by using a basis set as the one defined by Eq. (38) which depends on a non-linear parameter $\{$. Then, all matrices in the secular equation (26) are functions of this parameter and so are the eigenvalues (see Fig. 2).

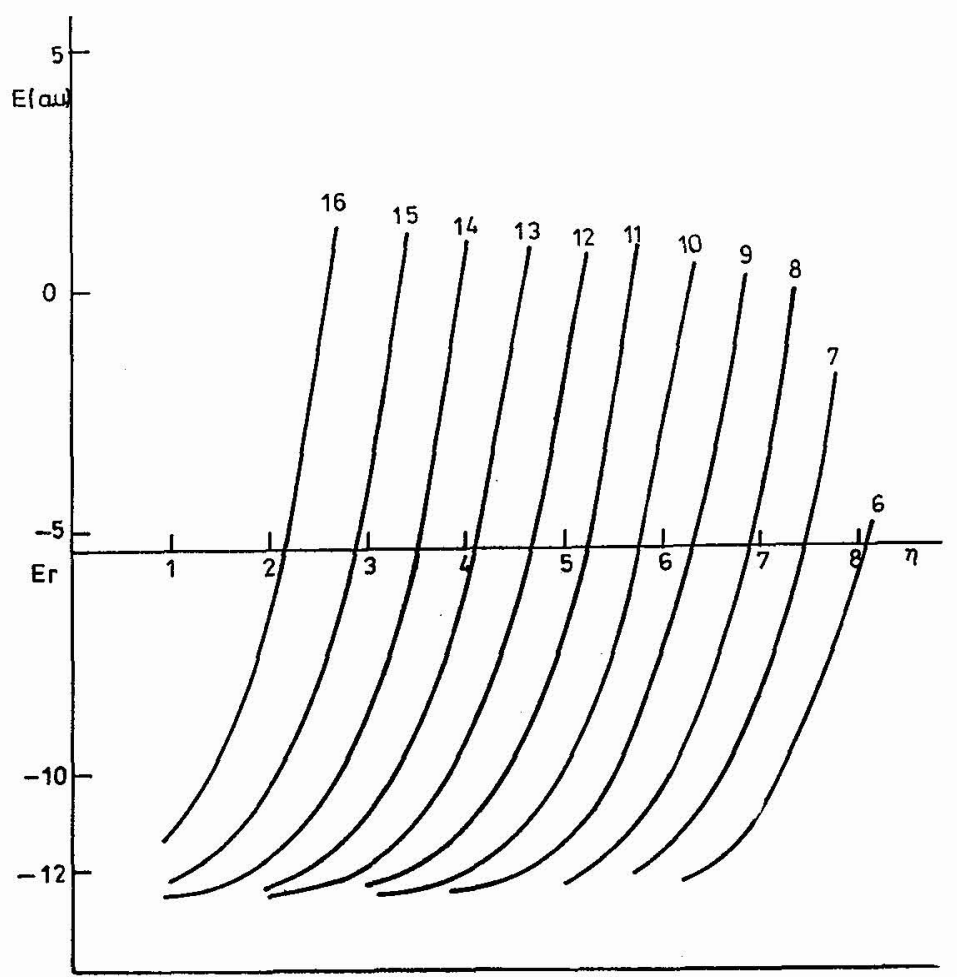

F18. 2 - Behavior of the eigenvalues of HFis as a function of the nonlinear parameter $\eta$.

Therefore we only need to chose this parameter by inverse interpolation so that $E_{m}=E_{1} a$. Figure 2 clearly shows that the resonance condition can be achieved for different values of $n$ and this fact permits to gauge the accuracy of our approach, in the sense that densities of states evaluated at neighbour values of $n$ should yield equal values for the width.

At this point it is worth to summarize the main steps of our procedure.

1) Ve carry out a CI calculation for the effective Hamilonian (14) using configurations built from a fintte basis of STO's or GTO'S. For twoeleotron systems, $V_{m}$ is zero, while for those systems consisting of a core and two valence electrons $V_{m}$ is a model potential $/ 29 /$ which accounts for core-valence effects. We take it to be of the single form /30-32/:

$$
V_{M}=\left(-2 / r_{i}\right)\left(1-\alpha r_{\text {s. }}\right) e^{-a \alpha r_{1}}
$$

where the parameter $\alpha$ is chosen by fitting the exact energy of the lowest valence state of the ionized system which has the same symmetry as the resonant state of interest. In all cases $M$ was taken to be 100 a. $u$. in Eq (16) and it was checked that increasing up to $1000 \mathrm{a}, \mathrm{u}$. did not change 
our data. Thus we obtain approximations to the (valence and) closed channel wave functions $\phi_{n}$ and to their energies $E_{m}$,

2) For each projector $P_{\kappa}$ (Eq, (8)) we carry out a CI calculation in a finite basis of Lfintegrable functions spanning a manifold projected onto by $P_{x}$. This is equivalent to include in each configuration one of the hydrogenic orbitals $\varphi_{x}$ entering the definition of $P_{x}$ (See Eq. (3)). For instance, if we are interested in evaluating (31,31') resonances of He-like systems, $\varphi_{\infty}$ is either of the 1s, 2s, 2p.4, 2p.y and $2 p o$ hydrogenic orbitals of the corresponding ionized system. We then obtain Lwintegrable approximations, $x \mathrm{~m}$, to the continuous wave functions, $X \mathrm{E}$ associated to each open channel.

3) A non-linear parameter is varled in the basis set used in step 2) to achieve, by inverse interpolation, the degeneracy condition between discrete and continuum components.

4) the density of states in Eq. (36) is evaluated using Eq. (41)

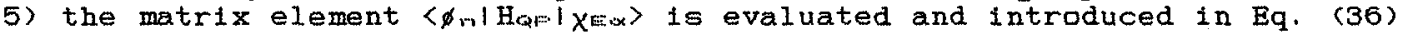
together with the corresponding value of $\rho$, to evaluate the partial width to that continuum. The total width of the autoionizing state of interest then follows from Eq. (9).

\section{7 - ILIUSTRATIONS}

We shall illustrate the performance of our theoretical approach for:

a) resonant states of He-like systems lying above the first or above several ionization thresholds. In particular, we shall present results for the first resonant states of 'P symmetry above the $n=1$ threshold of Helium and the first two (31,31') ":P resonant states af Nst whase energies lie above several lonization limits.

b) resonant states of Be-like systems; more specifically we shall present the energy positions and widths for the (1s:3131') ', $\mathrm{s}$ resonant states of $\mathrm{C}=-$ and $\mathrm{Ne} \mathrm{e}^{\mathrm{T}}$ ions, which are of great experimental interest $/ 35-39 /$.

c) molecular resonances of two-electron homo- and heteronuclear systems; in particular we shall show how the pseudopotential approach yields energy positions for molecular resonances for the whole range of internuclear distances, and we shall also present some widths for the range of very short internuclear distances where one-center expansions are sufficiently accurate to describe the molecular wave function.

\section{1 - He-LIKE SYSTEMS}

\section{1 .1 - RESONANCES UNDER $n=2$}

To obtain the positions and widths of the first ten ip resonant states af He above $n=1$, we have carried out a direct $C I$ calculation for the effective Hamilonian (12) with $\mathrm{Vm}_{m} \equiv 0$ and $M=100 \mathrm{a}, \mathrm{u}$.

Table 1 - Resonance energies (A) and widths (B) for the 'P states of He above the $n=1$ threshold. a) Feshbach-pseudopotential approach; b) conventional Feshbach method $/ 18 /$.

\begin{tabular}{llllll}
\hline A) & $-E$ (a. u.) & \multicolumn{3}{c}{$B$ ) $\Gamma$ (eV) } \\
\hline & $a)$ & b) & & $a)$ & b) \\
\hline$(2,2 a)$ & 0.69200 & 0.69213 & $(2,2 a)$ & $3.82(-2)$ & $3.74(-2)$ \\
$(2,3 b)$ & 0.59730 & 0.59730 & $(2,3 b)$ & $1.46(-4)$ & $1.43(-4)$ \\
$(2,3 a)$ & 0.56376 & 0.56379 & $(2,3 a)$ & $8.60(-4)$ & $8.45(-3)$ \\
$(2,3 c)$ & 0.54701 & 0.54701 & $(2,3 c)$ & $3.12(-4)$ & $3.03(-5)$ \\
$(2,4 b)$ & 0.54613 & 0.54613 & $(2,4 b)$ & $2.12(-5)$ & $2.10(-5)$ \\
$(2,4 a)$ & 0.53423 & 0.53425 & $(2,4 a)$ & $3.68(-3)$ & $3.62(-3)$ \\
$(2,4 c)$ & 0.52755 & 0.52755 & $(2,4 c)$ & $1.33(-5)$ & $1.28(-5)$ \\
$(2,5 b)$ & 0.52711 & 0.52711 & $(2,5 b)$ & $1.03(-5)$ & $1.03(-5)$ \\
$(2,5 a)$ & 0.52141 & 0.52142 & $(2,5 a)$ & $1.95(-3)$ & $1.91(-3)$ \\
$(2,5 c)$ & 0.51787 & 0.51787 & $(2,5 c)$ & $9.27(-6)$ & $8.60(-6)$ \\
\hline
\end{tabular}


The corresponding configurations were built from the basis set of STO's of Ref. 118/. The corresponding discretized continuum wave function was obtained by salving the secular equation (26) in the basis of configurations described in Ref, /18/. The results obtained /20/ have been summarized in Table 1 where comparison with values obtained using the conventional Feshbach method $/ 18 /$ is very encouraging.

\section{1 .2 - RESONANCES LYING ABOVE SEVERAL THRESHOLDS}

Our results /21/ for positions, total and partial widths for the first two (3131') $1, \Rightarrow P$ resonant states of $N^{5+}$ whose energies lie above three ionization limits ( $15,25,2 p$ ) are presented in Table 2 , along with results obtained with the conventional Feshbach method $/ 19,22 /$.

Table 2 - Resonance energies and widths for the 'P states of $N^{5+}$ above $n=$ 2 threshold, a) Feshbach-pseudopotential approach. b) Conventional Feshbach method $/ 19,22 /$

\begin{tabular}{lll}
\hline & $-E($ a. u. $)$ & \\
\hline & $a)$ & $b$ ) \\
\hline $1^{1} P^{0}$ & 5.02844 & 5.020 \\
$2^{1} p^{0}$ & 4.69834 & 4.720 \\
\hline & $\Gamma(\mathrm{eV})$ & \\
\hline $1^{1} p^{0}$ & $4.00(-1)\left(^{* *}\right)$ & $4.38(-1)$ \\
$2^{1} p^{0}$ & $1.13(-1)\left(^{* *}\right)$ & $1.14(-1)$ \\
\hline
\end{tabular}

(**) Partial widths:

\begin{tabular}{lllll}
\hline & $\Gamma_{1 \varangle \mathrm{p}}$ & $\Gamma_{\text {2xpp }}$ & $\Gamma_{2 p \mathrm{~d}}$ & $\Gamma_{2 p \mathrm{~d}}$ \\
\hline $1^{1} p^{0}$ & $3.37(-3)$ & $1.25(-1)$ & $1.93(-1)$ & $7.78(-2)$ \\
$2^{1} p^{0}$ & $1.67(-3)$ & $4.31(-3)$ & $5.63(-3)$ & $1.02(-1)$ \\
\hline
\end{tabular}

Overall agreement is very good, just as for resonances lying above a single ionization threshold. In this respect it is worth stressing again that conventional. Feshbach calculations are, for these cases, much more involved from the computational point of view and much more time consuming.

The basis sets used for both the discrete and the continuum wave function are given in Table 3 .

Table 3 - STO basis sets and configurations used in the calculation of closed- and open-channel wave functions of 'P symetry. The definition for each STO is: $\varphi_{m}\left(n, l, m, \zeta_{i}, r\right)=N_{i} r^{n-1} e^{-\zeta_{i}(Z) r} Y_{i}^{m}(\theta, \phi)$.

Closed-channel wave function $\left(^{*}\right)$

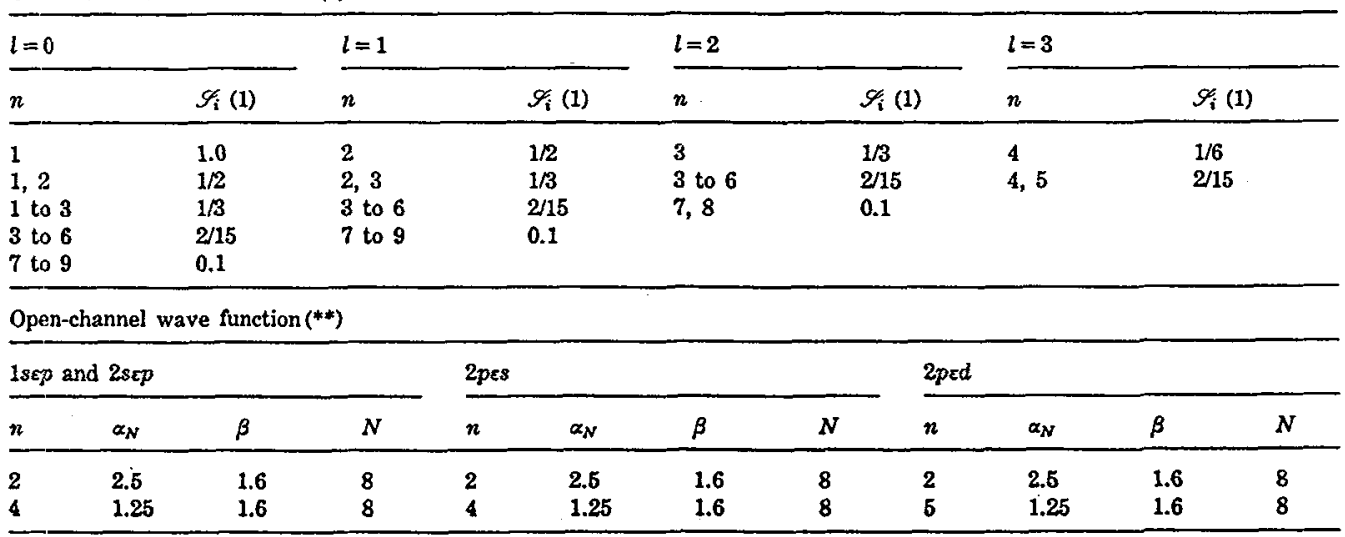

(*) Configurations included: All $n s n^{\prime} p$ with $n \leqslant 3$ or $n^{\prime} \leqslant 4$; all $n p n^{\prime} d$ with $n<5$; all $n d n^{\prime} f$ with $n<6$.

(**) Configurations included: Full CI 


\section{2 - Be-LIKE SYSTEMS}

In order to obtain the positions and widths of (16:3131\%) , ap resonances of $\mathrm{C}=+$ and $\mathrm{Ne}$ - the first step is to adjust the model potential of Eq. (42) so as to reproduce the exact energy of the lowest $\mathrm{s}$ states of $\mathrm{C}^{3+} \alpha=$ 3.7300) and $\mathrm{Ne}^{\mathrm{T}+}(\alpha=6.3943)$, respectively. The secular equation for the effective hamiltonian (12) was then set up in a representation of configurations built from the basis set of STO's given tn Table 4. This table also contains the sTo basis employed to solve the corresponding open channel equations (26).

Table 4 - STO basis sets and configurations used in the calculation of closed- and open-channel wave functions of , ap symetry of Be-like systems

\begin{tabular}{|c|c|c|c|c|c|c|c|}
\hline \multicolumn{8}{|c|}{$\begin{array}{l}1,3 p \text { states } \\
\text { Discrete }\end{array}$} \\
\hline & & & & & & & \\
\hline$n$ & $\zeta_{4}$ & $n$ & $\xi_{i}$ & $n$ & $S_{i}$ & $n$ & $\xi_{i}^{\prime}$ \\
\hline $1-3$ & $\frac{1}{3}$ & $2-3$ & $\frac{1}{3}$ & 3 & $\frac{1}{3}$ & 4 & $\frac{1}{4}$ \\
\hline $4-6$ & $\frac{1}{4}$ & $4-6$ & $\frac{1}{4}$ & $4-6$ & $\frac{1}{4}$ & $5-7$ & $\frac{1}{5}$ \\
\hline $7-9$ & $\frac{1}{7}$ & $7-9$ & $\frac{1}{7}$ & $7-8$ & $\frac{1}{7}$ & & \\
\hline
\end{tabular}

Configurations: All nsn'p with $n \leq 3$ or $n^{\prime} \leq 3$

All $n p n^{\prime} d$ with $n^{\prime} \leq 7$

All $n d n^{\prime} f$

\begin{tabular}{cccccccccccc}
\multicolumn{1}{c}{} & \multicolumn{1}{c}{ Continuum } \\
$n$ & $a_{N}$ & $\beta$ & $N$ & $n$ & $\alpha_{N}$ & $\rho$ & $N$ & $n$ & $a_{N}$ & $\rho$ & $N$ \\
\hline 2 & 2.5 & 1.6 & 8 & 2 & 2.5 & 1.6 & 8 & 3 & 2.5 & 1.6 & 8 \\
4 & 1.25 & 1.6 & 8 & 4 & 1.25 & 1.6 & 8 & 5 & 1.25 & 1.6 & 8 \\
\hline
\end{tabular}

Our results $120 /$ for positions, partial and total widths are presented in Table 5. In this case there are no conventional Feshbach results to compare with. However, for the particular case of 'P resonance positions comparisson with the experimental outcomes of Mack /35/ is very good, once our results have been corrected to take into account the post collisional interaction shift which affects the experimental outcomes $/ 20 /$.

Table 5 - Resonance energies and total and partial widths for "ap states of berylliumlike systems above the 1s:2p threshold.

\begin{tabular}{|c|c|c|c|c|}
\hline \multirow{2}{*}{\multicolumn{5}{|c|}{$\stackrel{I^{\prime} P}{-E(\text { (a.u.) }}$}} \\
\hline & & & & \\
\hline $\mathrm{C}^{2+}$ & 1.621883 & 1.433512 & 1.684652 & 1.513232 \\
\hline $\mathrm{Ne}^{6+}$ & 6.828589 & 6.387434 & 6.964866 & 6.577623 \\
\hline \multicolumn{5}{|c|}{$\Gamma(\mathrm{eV})$} \\
\hline $\mathrm{C}^{2+}$ & 0.22815 & $0.66209(-1)$ & $0.76196(-1)$ & $0.37109(-1)$ \\
\hline $\mathrm{Ne}^{6+}$ & 0.30392 & 0.14453 & $0.89977(-1)$ & $0.51413(-1)$ \\
\hline
\end{tabular}

Partial widths:

\begin{tabular}{cll}
\multicolumn{1}{c}{$\Gamma_{\text {2up }}$} & \multicolumn{1}{c}{$\Gamma_{2 \rho s}$} & \multicolumn{1}{c}{$\Gamma_{2 p \text { d d }}$} \\
\hline $0.72785(-1)$ & 0.13233 & $0.23033(-1)$ \\
$0.18307(-2)$ & $0.43199(-2)$ & $0.60058(-1)$ \\
$0.30691(-1)$ & $0.43522(-1)$ & $0.19825(-1)$ \\
$0.35218(-2)$ & $0.91620(-2)$ & $0.24425(-1)$ \\
0.10340 & 0.15578 & $0.44736(-1)$ \\
$0.19899(-2)$ & $0.11379(-1)$ & 0.13116 \\
$0.41664(-1)$ & $0.43476(-1)$ & $0.48370(-2)$ \\
$0.35188(-2)$ & $0.15963(-1)$ & $0.31931(-1)$
\end{tabular}




\section{3 - MOLECULAR RESONANCES}

\section{3 .1 - POSITIONS}

We have chosen as a suitable model system for our illustration the i $\Sigma$ resonant states of the LiHe ${ }^{3-m}$ quasimolecule which are involved in LI:- + He (Is:) collisions. These states lie above two Rydberg series $\left(\mathrm{Li}^{+}(1 \mathrm{snl})+\right.$ $\left.\mathrm{He}^{-+}\right\}$and $\left\{\mathrm{Liz} \cdot(1 \mathrm{~s})+\mathrm{He}^{+}(\mathrm{n} 1)\right)$, both converging to the first ionization linit LiHen-(1so). For the sake of consistency the corresponding effective Hamitonian (12) was diagonalized in a configuration basis identical to that employed in the conventional Feshbach calculations of Ref. /40/, The results /23/ are plotted in Fig. 3. The agreement between these data and those of Ref. $/ 40 /$ is so good that both curves are indistinguishable from each other in the whole range af internuclear distances considered.

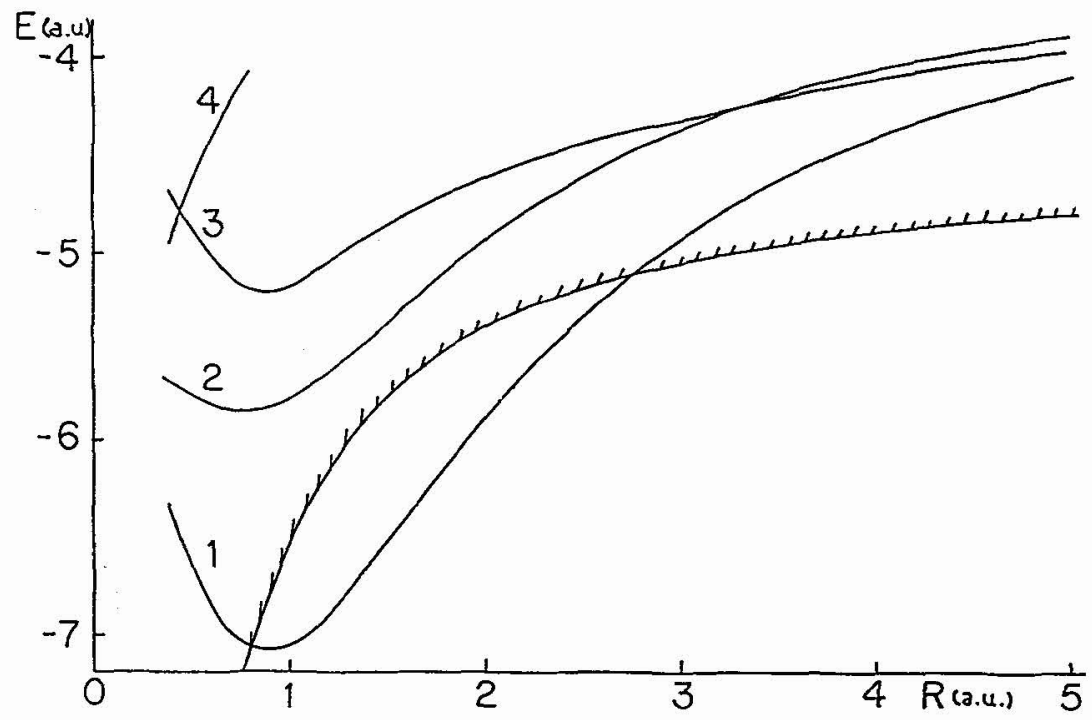

Fig. 3 - Electronic energies of the autoionizing states of the LiHe quasimolecule obtained with either the Feshbach or pseudopotential methods (both sets of values are indistinguishable in this figure)

We have also evaluated the $1^{\prime} \Sigma-2^{\prime} \Sigma$ radial coupling which determines the mechanism of charge exchange in $\mathrm{Li}^{\mathrm{a}}+\mathrm{He}(1 \mathrm{~s})$ collisions.

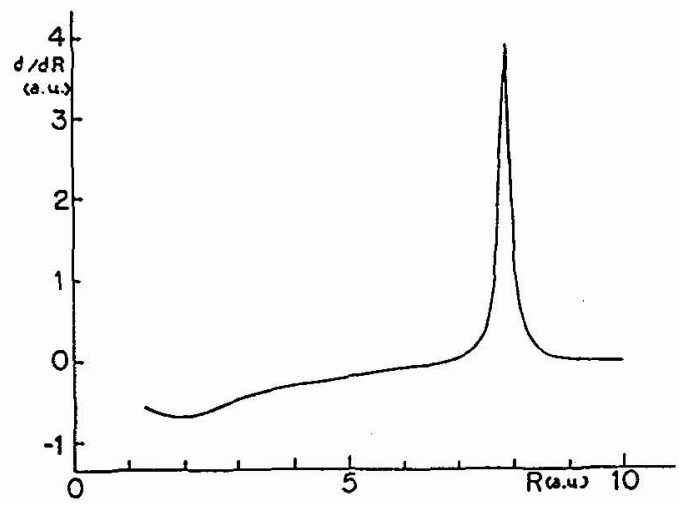

Fig. 4 - Radial coupling between the $1^{\prime} \Sigma$ and $2^{\prime} \Sigma$ resonant states of the LIHew quasimolecule obtained with either the Feshbach or pseudopotential methods (both sets of values are indistinguishable in this figure) 
As for the energy positions, the coupling given in Fig, 4 is practically indistingulshable from that obtained in Ref. /40/ using the conventional Feshbach method.

\section{3 .2 - WIDTHS}

We have employed the scheme presented in Section 6 to calculate the width for the region of very small internuclear distances, of the is resonant states of $\mathrm{LiHe}^{\mathrm{*} *}$ and $\mathrm{HeH}^{*}$ quasimolecules which are correlated in the UA limit to 'P(2s2p), as well as for the first ' ${ }^{*}$, resonant state of $H a$ and Hea as typical benchmark homonuclear systems.

In the region of small internuclear distances one-center expansions for $\phi_{n}$ are a sensible choice, the only problem being how to chose their origin in the heteronuclear case. We have adopted as the intuitively most reasonable cholce the center of nuclear charge. For both LiHe a- and $\mathrm{H}^{-2}$ the same basis sets of Ref. /27/ have been used. The corresponding open channel wave functions were approximated as 1 inear combinations of configurations built from the set of STO's of Ref, /41/ and also centered at the center of nuclear charge.

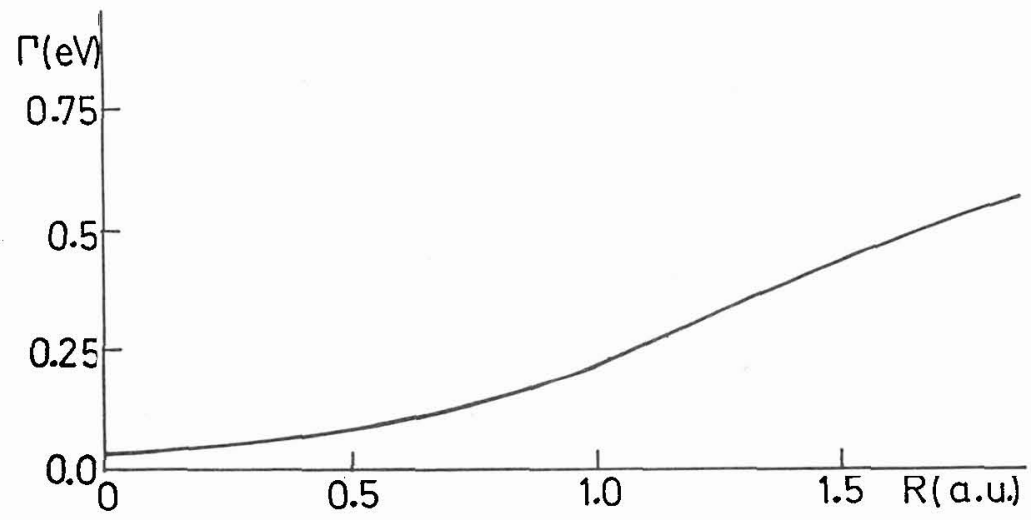

F1g. 5 - Variation of the width of the first ' $\Sigma$ w resonant state of $H_{a}$ with the internuclear distance.

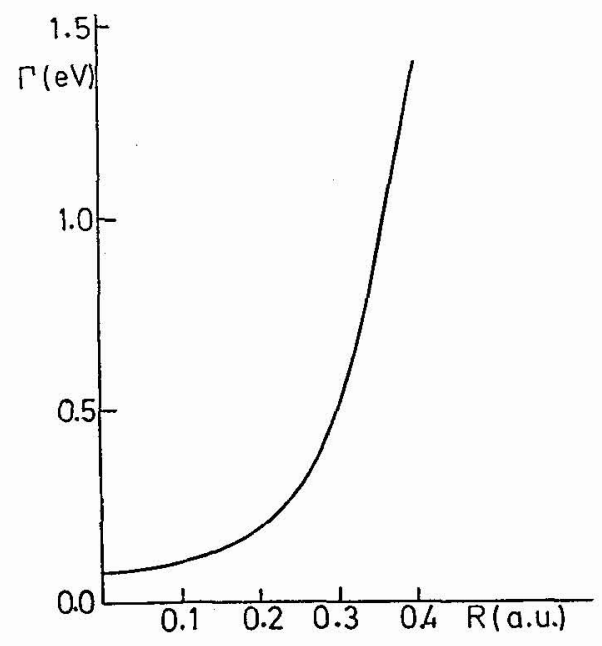

Fig. 6 -Variation of the width of the ' $\Sigma$ resonant state correlated in the UA limit to 'P (2s2p) of LiHe 
For the sake of conciseness we shall discuss only the widths obtalned for LiHe $3+$ and $H_{*}$ molecules /27/ which are presented in Figs. 5 and 6 respectively. Both increase considerably with $R$. This can be understood 127) by analyzing the shape (See Fig. 7) of the $\epsilon$ p continuum orbital which describes the outgoing electron.

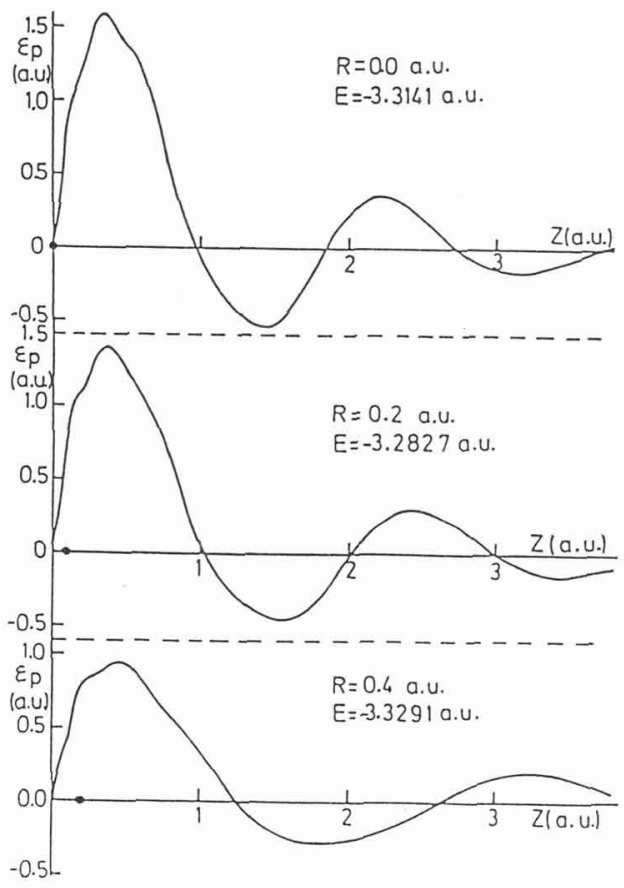

Fig. 7 - Evolution with the internuclear distance of the shape of the $E \mathrm{p}$ orbital which describes the outgoing electron of the He: molecule

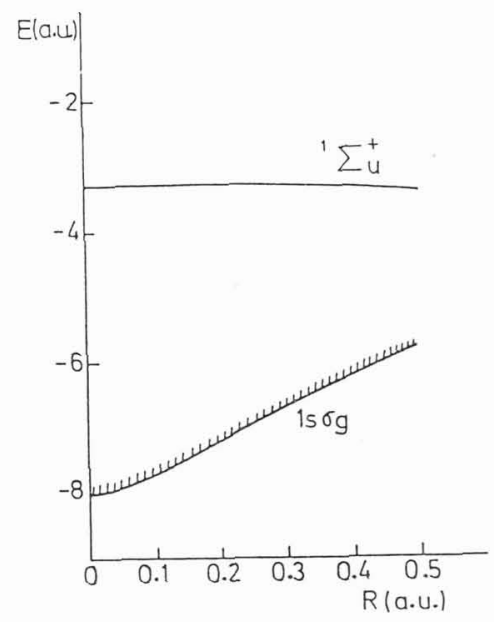

Fig. 8 - Variation of the energy of the first ' $\Sigma_{w^{+}}$resonant state Hez-k and of Hea (1sra) lonization limit as function of the internuclear distance. 
Fig. 7 shows that, as R increases, this orbital presents less oscillations in the molecular region and as a consequence its interaction with the closed channel component becomes more effective and greater the width. This increase of the average wavelength of the continuura orbital is a consequence of the fact that, as $R$ increases, the energy difference between the resonance and the ionization threshold (See Fig. 8) decreases very rapidly and so the kinetic energy of the outgaing electron.

As in atomic cases, there is full agreement between these results and those obtained from conventional Feshbach calculations /27/.

Acknowledgements

This research was partially supported by the CAICYT project No. $890 / 84$

\section{REFERENCES}

11 Andersen, T., Bentzen, S.M. and Poulson, 0., Phys. Scr. 22P (1980) 119 /2/ Cederquist, H., Kislelinski, M, and Mannervik, S., J. Phys. B 16 (1983) 1479

/3/ Pochat, A, Tweed, R.J., Dorttch, M. and Paresse, J., J, Phys. B 15 (1982) 2269

14/ Hulse, R.A., Nucl. Tech. Fusion 3 (1983) 259

15/ Bruch, R., Altick, P.L., Trabert, E., and Heckmann, P.H., J. Phys. B 17 (1984) 1655

16/ Bruch, H. , Phys, Scr. 26 (1983) 381

17/ Van Sprong, H., Brangersma, H.H. and de Heer, F.J., Chem. Phys. Lett 65. (1979) 55

18/ Morgenstern, R., Nieuhaus, A, and Zimmerman, G., J. Phys. B 13 (1980) 4811 ; Boskamp, E., Morgenstern, R. and Nieuhaus, A., ibid. 15 (1982) 4577

19/ Cocke, C.L., Dubois, R., Gray, T.J., Justiniano, E. and Can, C., Phys Rev, Lett, 46 (1981) 1671

l10/ Bliman, S., Hitz, D., Jacquot, B., Harel, C. and Salin, A., J. Phys B 16 (1983) 2849 ; Bliman, S., Bonnefoy, M., Bannet, J.J., Dousson, S. Fleury, A., Hitz, D, and Jacquat, B., Phys. Scr. I3 (1983) 63

111 Itoh, A., Schneider, T., Schiwietz, S, Roller, Z, Platten, H, Notte, G., Schneider, D. and Stolterfoht, N., J. Phys. B 16 (1983) 3965 12, Cornille, M., Dubau, J., Bely-Dubau, F., Bliman, S,, Hitz, D., Mayo M. Bonnet, J.J., Bonnefoy, M., Chassevent, M. and Fleury, A., J. Phys. B 19 (1986) L393

/13/Feshbach, H., Ann. Phys, (N.Y.) 5 (1958) 357 ; ibid. 19 (1962) 287

/14/ Lipsky, L., Anania, R, and Conneely, M. J. At. Data 20 (1977) 127

/15/ Conneely M.J. and Lipsky, L., J. Phys, B 11 (1978) 4135

16/ Macías, A. and Riera, A., Phys. Lett, A103 (1984) 37?

$117 /$ Macías, A. and Riera, A., Chem. Phys. Lett. 117 (1985) 42

118/ Macias, A. and Riera, A., Phys. Lett. A119 (1986) 28

/19/ Bachau, H., J. Phys. B 17 (1984) 1771

l20/ Martín, F., Mó, O., Riera A. and Yañez, M., Phys, Rev. A 〈in press〉

121 Martín, F., Mó, O., Riera A. and Yáñez, M., Europhys. Lett. 4 (1987) 799

122/ Martin, F, Riera, A, Yáñez, M. and Bachau, H., J. Phys, B 21 (1988) 2261

l23/ Martín, F., Mó, O., Riera, A, and Yẫez, M., J. Chem. Phys. 87 (1987) 6635

/24/ Phillips, J,C. and Kleinman, I., Phys, Rev. 116 (1959) 287

125, Macias, A., Martín, F., Riera, A. and Yañez, M., Phys. Rev. A 36 (1987) 4179

/26/ Macias, A., Martín, F., Riera, A. and Yáñez, M., Phys. Rev. A 36 (1987) 4187

/27/ Macías, A., Martin, F., Riera, A. and. Yáñez, M., Phys. Rev. A 36 (1987) 4203

/28/ O. Nalley, T.F. and Geltman, S., Phys. Rev. 137 (1965) A1344

129/ Heeks, J.D., Hazi, A. and Rice, S.A., Adv. Chem Phys, 16 (1969) 283

Peach, G., Comm. At. Mal. Phys, 11 (1982) 101 ; Bonffacic, V. and Huzinaga S., J. Chem. Phys. 60 (1974) 2779 and references therein.

$130 /$ Mó, O., Riera, A. and Yáfiez, M., Phys, Rev. A 31 (9185) 3977

131 Mendizábal, R., Mó, O., Riera, A. and Yarrez, M., J. Mol. Struct Theochem 150 (1987) 150

(32/ Mó, O, and Riera, A., J, Mol Struct. Theochem. 164 (1988) 135 
133/ Macias, A., Martín, F., Riera, A. and Yariez, M., Int. J, Quant. Chem XXXIII (1988) 279

/34/ Macias, A. and Riera, A., J, Phys. 46 (1985) 535

/35/ Mack, M., Nucl. Instr. Meth. B23 (1987) 74

/36/ Mack, M. and Niehaus, A., Nucl. Instr. Meth. B23 (1987) 116

137/ Mack, M. and Niehaus, A., Abstracts 15th Int. Conf. on the Physics of Electronic and Atomic Collisions. Eds. J. Geddes, H.B. Gilbody, A.E Kingston, C.J. Latimer and H.J.R. Walters, p 567, Brighton (1987)

138/ Bordenave-Montesquieu, A, Benoit-Cattin, P., Boudjema, M., Gleizes A. Dousson, $S$, and Hitz, D., Abstracts 15th Int, Conf, on the Physics of Electironic and Atomic Collisions. Eds. J. Geddes, H.B. Gilbody, A.E Kingston, C.J. Latimer and H.J.R. Walters, p 551, Brighton (1987)

139/ Bordenave-Montesquieu, A., Benoit-Cattin, P., Boudjema, M. and Gieizes, A., Book of invited papers. 15th Int. Conf. on the Physics of Electronlo and Atomic Collisions. Eds. H. B. Gilbody, W. R. Newell, F.H. Read and A.C.H, Smith, North-Holland, Amsterdam (1988), p 643

140/ Martín, F., Riera, A, and Yânez, M., J. Chem. Phys. 84. (1986) 5412

141/ Macias, A., Martín, F., Riera, A, and Yafiez, M., Phys. Rev, A 36 (1987) 4187 